\title{
Hyperalgesic Lumbosciatica Symptomatic of a Spinal Schwannoma: A Case Report
}

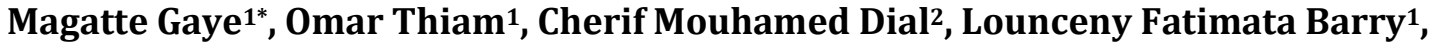 \\ Mohameth Faye1, Nantene Doumbia1, Youssoupha Sakho' \\ ${ }^{1}$ Department of Neurosurgery, Hopital General Grand Yoff, Dakar, Senegal \\ ${ }^{2}$ Department of Pathology, Hopital General Grand Yoff, Dakar, Senegal \\ Email: *magou762003@yahoo.fr
}

How to cite this paper: Gaye, M., Thiam, O., Dial, C.M., Barry, L.F., Faye, M., Doumbia, N. and Sakho, Y. (2022) Hyperalgesic Lumbosciatica Symptomatic of a Spinal Schwannoma: A Case Report. Open Journal of Modern Neurosurgery, 12, 61-66. https://doi.org/10.4236/ojmn.2022.122006

Received: January 16, 2022

Accepted: February 26, 2022

Published: March 1, 2022

Copyright $\odot 2022$ by author(s) and Scientific Research Publishing Inc. This work is licensed under the Creative Commons Attribution International License (CC BY 4.0).

http://creativecommons.org/licenses/by/4.0/

\begin{abstract}
Introduction: Spinal schwannomas also known as neurinomas are often benign slow growing lesion that may develop from Schwann cells of the spinal roots, it is a nerve sheath tumor. The authors reported a case of a patient presenting a hyperalgesic lumbosciatica symptomatic of a spinal schwannoma. Observation: A 36-year-old female patient, with a history of asthma under treatment was admitted to our department because of one year lasting of an intermittent fashion bilateral L5 hyperalgesic lumbosciatica. The initial examination has shown back muscles contractures and a segmental deficit of the right lower limb in L5 and S1, but no genital or sphincter disorders were noted. The lumbosacral CT scan was without particularity but the magnetic resonance imaging (MRI) revealed an intradural, extra medullary lesion at the level of L1-L2. A monobloc resection of the lesion was done. The follow-up is good. Pathology concluded in schwannoma WHO grade I classification. Conclusion: Lumbar neurinoma that grows slowly is most often manifested by a radiculalgia often hyperalgesic and disabling. MRI is the examination of choice to make the diagnosis and complete removal is possible.
\end{abstract}

\section{Keywords}

Lumbosciatica, MRI Spinal Tumor, Neurinoma, Surgery

\section{Introduction}

Schwannomas or spinal neurinomas are benign tumors that develop from the Schwann cells of the spinal roots. The term neurinoma was proposed in 1910 by VEROCAY to designate an encapsulated tumor developed on a root or a peripheral nerve precisely from the Schwann sheath [1]. They represented about $30 \%$ of intra spinal tumors, $80 \%$ of which are distributed all along the spinal 
cord and $20 \%$ were on the cauda equina. Neurinomas are most often sporadic but can be familial belonging to type II neurofibromatosis. The posterior sensory root is usually the bearer of the lesion that explains the frequency of radiculalgia that often reveals the pathology. Diagnosis has been facilitated by the development of neuroimaging, essentially with MRI, which has brought about a real revolution in topographical diagnosis, surgical planning and monitoring [2]. The objective of this work is to report a case of a hyperalgesic lumbosciatica symptomatic of intra spinal neurinoma discovered on spinal MRI in a patient coming with a normal lumbosacral CT scan. At one month post-operative follow up, she made a rapid full recovery.

\section{Case Report}

A 36-year-old female patient, with a history of asthma under treatment, was seen in consultation for bilateral hyperalgesic lumbosciatica of L5 topography, arising predominantly on the right limb, lasting almost for one year with sometimes by relapses and remissions. Any notion of intermittent radicular claudication was found.

On admission physical examination is critical because of the status of hyperalgesia. Walking was difficult, but possible with help. We noted a lumbar spinal syndrome with contracture of the para spinal muscles and a positive bell sign in L5 and S1. The neurological examination showed a segmental deficit of the right lower limb rated at 3/5 in L5 and 4/5 in S1. There was no sensory or genitourinary disorder. The CT scan of the lumbosacral spine was within normal limits unless a slight disc protrusion in L3 - L4 and L4 - L5 on the right could be noted (Figure 1(a)). In addition, the dimensions of the canal were normal (Figure 1(b)) meaning no scalloping is present.

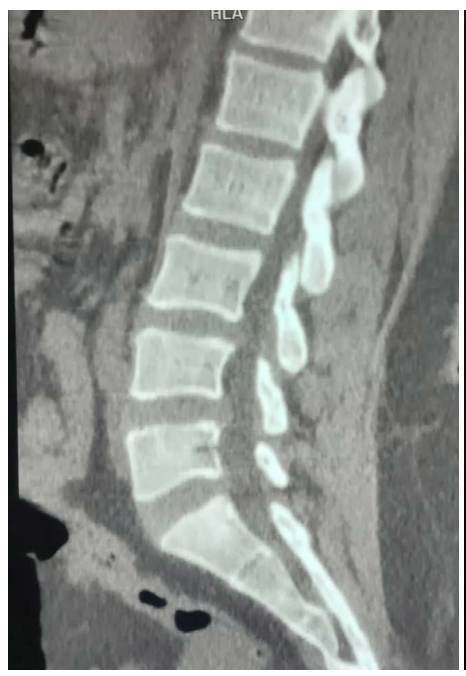

(a)

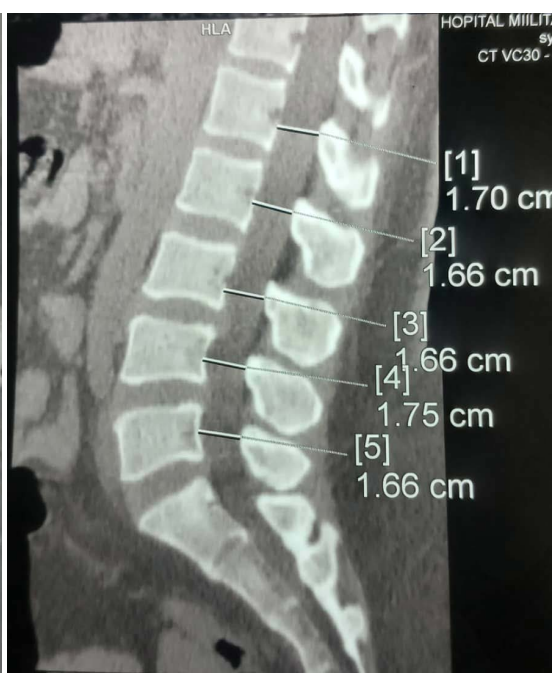

(b)

Figure 1. CT scan of the lumbosacral spine, sagittal reconstructions showing a disc bulge between L3-L4 and L4-L5 (a) with canal dimensions within the normal range (b), no scalloping is found. 
Because of the discrepancy between the symptomatology and the CT scan finding, lumbar sacral spine MRI was performed and shown an intra spinal ovoid lesion measuring a length $55.8 \mathrm{~mm}$ and width $19.3 \mathrm{~mm}$, located between $\mathrm{L} 1$ and L2. It was hypo intense in T1 sequence (Figure 2(a)) and heterogeneous hyper intense in T2 (Figure 2(b)). In axial view, the lesion is rounded shaped and eccentrically on the right side (Figure $2(\mathrm{c})$ ).

The patient underwent surgery, we realized complete laminectomy of L1, L2 and partial laminectomy of T12, dural sheath was enlarged and tense (Figure $3(\mathrm{a})$ ). Just after opening longitudinally the dura mater a firm grayish tumor lesion

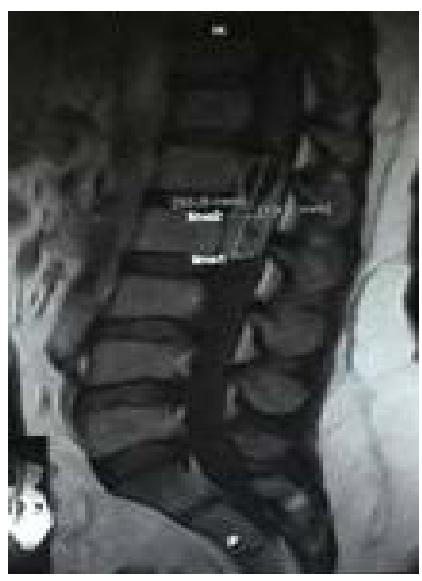

(a)

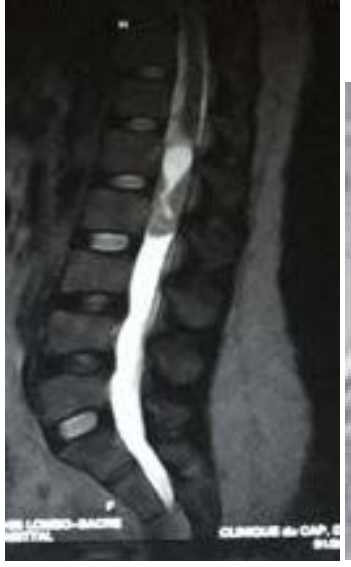

(b)

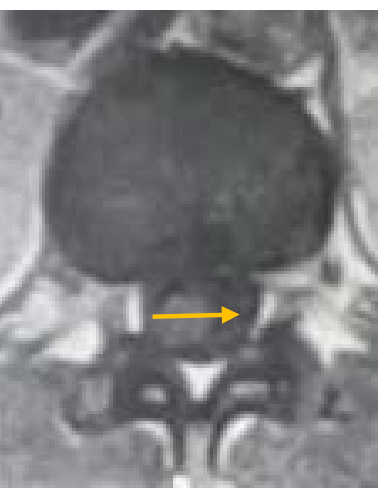

(c)

Figure 2. Lumbar MRI showing an intra spinal ovoid lesion going from the posterior edge of L1 to L2 in T1 (a) and T2 sagittal view (b). In axial view the lesion (yellow arrow) is rounded shaped and eccentrically on the right side (c).

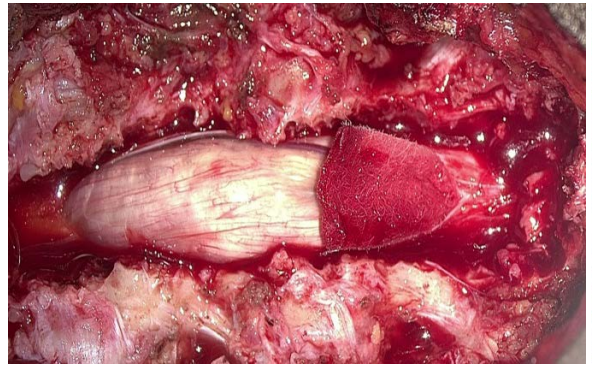

(a)

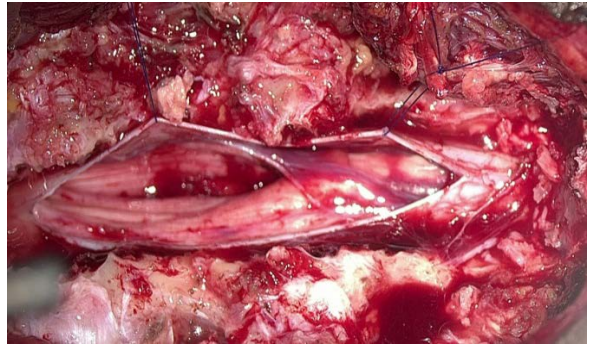

(c)

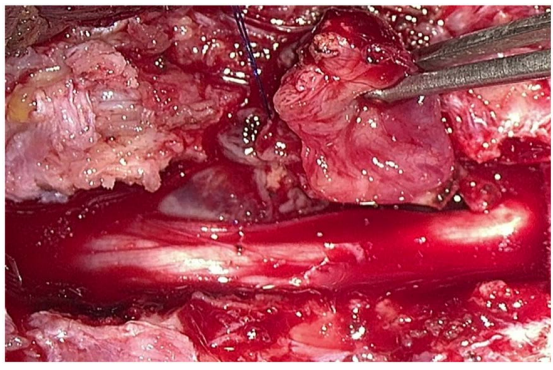

(b)

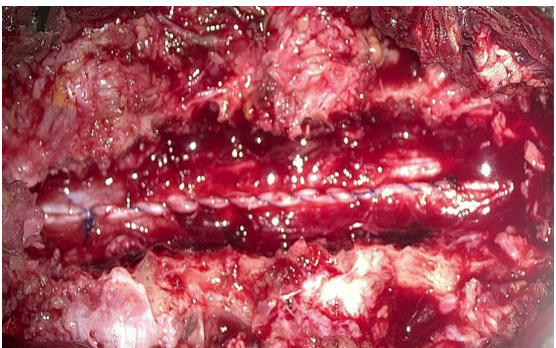

(d)

Figure 3. Different surgical steps: dural opening showing an enlarged spinal cord (a), tumor removal (b), aspect of the bed of the lesion (c), tight dural closure (d). 
attached to the root was seen (Figure 3(b)). Complete excision was performed and hemostasis was satisfactory (Figure 3(c), Figure 3(d)).

The postoperative course was uneventful, with a dramatic relief of pain in the symptoms at day 1 post surgery a neurological recovery was noted: the motor strength on the right was rated 4.

The patient was discharged on postoperative day 5 with a prescription for physical therapy. Pathology concluded to lesion with spindle cell proliferations arranged in a sheet. The tumor cells have elongated monomorphic nuclei with poorly restricted eosinophilic cytoplasm suitable with Grade I schwannoma (Figure 4).

The patient was reviewed in consultation at one month postoperatively, the surgical wound had healed and there was a complete disappearance of the radicular pain and neurological deficit. The neurological examination was strictly normal.

\section{Discussion}

Spinal schwannomas represent $30 \%$ of primary intra spinal tumors. The incidence of spinal schwannomas, according to recent studies, varies between 3 and 4 cases per 1,000,000 people per year with no clear difference according to gender [3].

Spinal schwannomas are usually intradural extra-medullary tumors, rarely intramedullary. Mostly the lesion is unique but sometimes it could be multifocal along the neuroaxis in such case a context of type 2 neurofibromatosis exists [4] [5]. Spinal neurinomas occur more often between 30 and 50 years old. Childhood presentation are rare. The cervical location is frequently encountered, followed by the thoracic location, with respectively $58 \%$ and $32 \%$. The lumbar location represents only $10 \%$ [4] [6].

The clinical presentation is insidious depending on the location of the lesion and its relationship within the spinal cord. Radicular the pain is often the starting point, like our patient who was suffering from a long lasting lumbosciatica

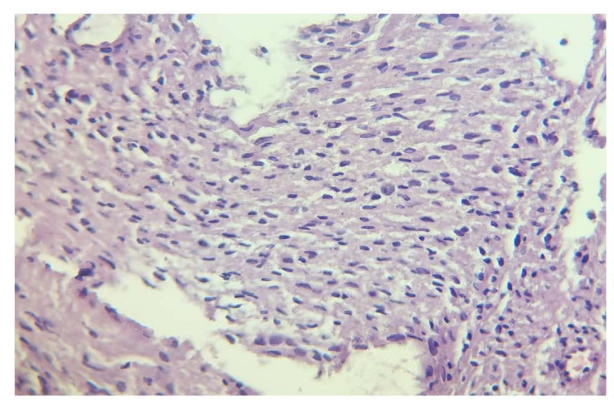

(a)

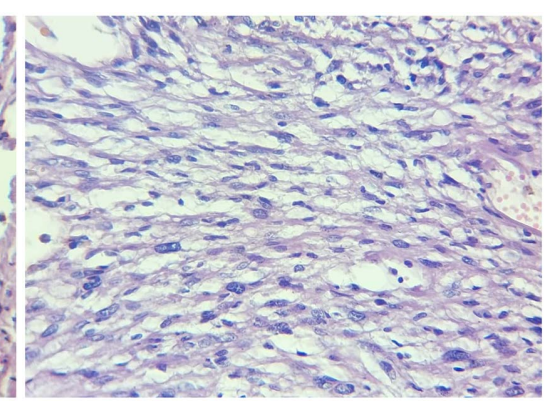

(b)

Figure 4. Histologic appearance in two areas of a benign schwannoma with spindle cell proliferations arranged in a sheet. The tumor cells have elongated monomorphic nuclei with poorly restricted eosinophilic cytoplasm. (a) 150 Hematoxylin eosin; (b) 200 Hematoxylin eosin. 
with episode of intractable pain. Motor, sensory deficit and sphincter disorders are possible. Some paradoxical clinical manifestations like an acute hydrocephalus have been described through the literature [4]. These symptoms are thought to be related on high rate of albumin into the cerebral spinal fluid consequences of tumor micro hemorrhage or of tumor obstruction of the subarachnoid spaces.

Spine CT scan sometimes demonstrated indirect canal compromise with the presence of scalloping of the posterior edge of a vertebral body, it is an imaging feature highly suggestive of intra spinal neurinoma. In our patient this aspect is not seen. Magnetic resonance imaging is a gold standard for the diagnosis of intra spinal lesions it allows a nice topographic analysis of the lesion. In T1 sequences, neurinoma appears usually iso intense surrounded by a hypo signal that correspond to the cerebrospinal fluid (CSF). In T2 the lesion is hyper intense and homogenous post gadolinium enhancing [2] [7]. In areas where MRI is not available, myelography combined with CT scan may be of interest in the diagnostic process.

Surgery is essential in the treatment of spinal schwannomas, its objective is to relief the neural structure from the tumor compression. The surgical resection of the neurinoma should be as complete as possible to avoid recurrence. The choice of the approach depends on the location and its relationship to the spinal cord. Total resection of intradural extra-medullary forms is possible, but total resection of intramedullary forms is challenging [8] [9].

A fast postoperative recovery has been noted in the literature [8] like our patient. Surgical site infections and spinal instability resulting from extensive laminectomy or pedicle resection could arise as a complication. Thus, posterior instrumentation may be indicated in these cases [3]. No complications were noted in our patient. The deficit resulting from the sacrifice of the bearing root is often well tolerated [6].

\section{Conclusion}

Lumbar spinal schwannoma is an under-diagnosed condition; sometimes radiculalgia without any signs of neurological compression may be the first presentation. Magnetic Resonance Imaging is the gold standard in the diagnosis of this lesion. Total excision of tumor mass could be done safely without any complication.

\section{Informed Consent}

Informed consent was obtained from the patient for publication of this manuscript and any accompanying images.

\section{Conflicts of Interest}

The authors declare no conflict of interest or any financial support.

\section{References}

[1] Alter, M. (1975) Statistical Aspects Cord Tumors. In: Klawans, H.L., Ed., Tumours 
of the Spine and Spinal Cord: Part I. Handbook of Clinical Neurology, American Elsevier, New York, 1-22.

[2] Idir, A.B.C., Doyon, D., Husson, B. and et Hurt, M. (1991) Pathologie tumorale de la moelle et des enveloppes chez l'adulte et chez l'enfant. Encycl. Med. Chir. (Paris-France), radiodiagnostic neurologie. Appareil Locomoteur, 31673a, 12.

[3] Emel, E., Abdallah, A., Sofuoglu, O.E., Ofluoglu, A.E., Gunes, M., Guler, B. and Bilgic, B. (2017) Long-Term Surgical Outcomes of Spinal Schwannomas: Retrospective Analysis of 49 Consecutive Cases. Turkish Neurosurgery, 27, 217-225.

[4] Himmiche, M., Joulali, Y., Benabdallah, I.S., Benzagmout, M., Chakour, K., et al. (2019) Shwannomes rachidiens: étude de series. The Pan African Medical Journal, 33, 199. https://doi.org/10.11604/pamj.2019.33.199.17921

[5] Li, P., Zhao, F., Zhang, J., Wang, Z., Wang, X., et al. (2016) Clinical Features of Spinal Schwannomas in 65 Patients with Schwannomatosis Compared with 831 with Solitary Schwannomas and 102 with Neurofibromatosis Type 2: A Retrospective Study at a Single Institution. Journal of Neurosurgery, 24, 145-154. https://doi.org/10.3171/2015.3.SPINE141145

[6] Celli, P., Trillo, G. and Ferrante, L. (2005) Spinal Extradural Schwannoma. Journal of Neurosurgery, 2, 447-456. https://doi.org/10.3171/spi.2005.2.4.0447

[7] Colosimo, C., Cerase, A., Denaro, L., Maira, G. and Greco, R. (2003) Magnetic Resonance Imaging of Intramedullary Spinal Cord Schwannomas. Journal of Neurosurgery, 99, 114-117. https://doi.org/10.3171/spi.2003.99.1.0114

[8] Conti, P., Pansini, G., Mouchaty, H., Capuano, C. and Conti, R. (2004) Spinal Neurinoma: Retrospective Analysis and Long-Term Outcome of 179 Consecutively Operated Cases and Review of the Literature. Surgical Neurology, 61, 34-43. https://doi.org/10.1016/S0090-3019(03)00537-8

[9] Varshney, R., Bharadwaj, P., Choudhary, A., Paliwal, P. and Kaushik, K. (2020) A Rare Case of Intramedullary Schwannoma at Conus Medullaris: A Case Report with Review of Literature. Surgical Neurology, 11, 454.

https://doi.org/10.25259/SNI_718_2020 